\title{
A Fast Time-Delay Calculation Method in Through-Wall-Radar Detection Scenario
}

\author{
Zhang $Q{ }^{1}{ }^{1}$, Lei Wentai ${ }^{1}$, Wang Qianzhe ${ }^{2}$ \\ ${ }^{1}$ School of Information Science and Engineering, Central South University, No. 932, Lushan South Road, Changsha, Hunan, P. R. China \\ ${ }^{2}$ Department of Scientific Research, Air Force Engineering University, No.1, Changle East Road, Xi'an, Shanxi, P. R. China
}

\begin{abstract}
In TWR (Through Wall Radar) signal processing procedure, time delay estimation is one of the key steps in target localization and high resolution imaging. In time domain imaging procedure such as back projection imaging algorithm, round trip propagation time delay at the path of "transmitter-target-receiver" needs to be calculated for each pixel in imaging region. In typical TWR scenario, transmitter and receiver are at one side and targets at the other side of a wall. Based on two-dimensional searching algorithm or solving two variables equation of four times, traditional time delay calculation algorithms are complex and time consuming, and cannot be used to real-time imaging procedure. In this paper, a new fast time-delay (FTD) algorithm is presented. Because of that incident angle at one side equals to refracting angle at the other side, an equation of lateral distance through the wall can be established. By solving this equation, the lateral distance can be obtained and total propagation time delay can be calculated subsequently. Through processing simulation data, the result shows that new algorithm can be applied effectively to real-time time-delay calculation in TWR signal processing.
\end{abstract}

\section{Introduction}

TWR is now widely used in various application scenarios, while it ensures that researchers obtain quantitative and qualitative information in the media [1]. The quantitative information is obtained by full wave transposition, including electromagnetic property. Qualitative information can be extracted from TWR echo to estimate properties such as size, shape, permittivity ect. of subsurface target by using inverse scattering imaging algorithms. TWR becomes a hot research area and a lot of achievements have obtained [2,3]. Time delay calculation is very important in time domain imaging. E.M.Johansson and J.E.Mast use linear approximation method to get the electromagnetic waves propagate timedelay [4]. Rappaport.C.M describes that the waves propagate with a wavefront shape that resembles a hyperbola when enter the medium, and a formula is provided to reduce the error between the approximate hyperbola and the exact shape [5]. Lei et al. present a new fast time-delay calculate method based on onedimensional searching and solving quartic equation [6]. Chen et al. give an approximate round-trip time delay algorithm to reduce the wall clutter [7]. Protiva et al. present a wall parameter estimation method that utilizes time-delay-only measurements of echoes backscattered from a wall [8]. However these methods are complex, time-consuming, and not applicable at real time imaging in TWR, so this paper proposes an improvement method to solve this problem.
In this paper, a fast time-delay calculation (FTD) is presented. By analyzing electromagnetic wave propagation path, we can get the lateral distance in the wall and total path, and then time delay can be calculated. Through processing simulation data, the results show that the algorithm can be applied effectively to time-delay calculation.

\section{Theoretical analysis}

A physical model of electromagnetic waves propagation in TWR application is shown in Fig. 1. Transmitter and receiver are located at point $\mathrm{A}$ and target is located at point B. An impulse signal is transmitted from transmitting antenna located at point $\mathrm{A}$ and reached point B after two times reflection at two sides of a wall respectively.

Electromagnetic waves propagate from the starting point $\mathrm{A}$ on the one side of the wall, and the incident point $\mathrm{C}$ is at the upper surface of the wall, then waves refract out of the wall on the lower side at point $\mathrm{D}$ after transmitting a distance in the wall, finally reach the receiving point B. ${ }^{\varepsilon_{1}}$ is the relative permittivity of the environment at both two sides of the wall, $\varepsilon_{2}$ is the relative permittivity of the wall, $d$ is the thickness of the wall. We define the top surface of the wall as $z=0, z$ is the vertical coordinate, the starting point's coordinate 
position is $\left(x_{a}, z_{a}\right)$, and the receiving point's coordinate position is $\left(x_{b}, z_{b}\right)$.

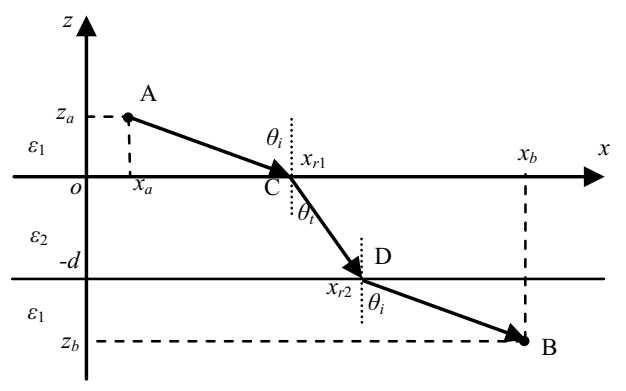

Figure 1. Electromagnetic waves propagation model.

\subsection{Traditional calculation method}

Traditional method for calculating electromagnetic wave propagation time-delay through wall is finding the minimum value of one-way propagation time-delay by two dimensional searching, shown below:

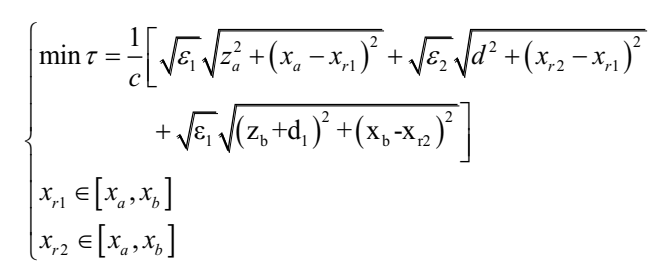

where $x_{r 1}$ and $x_{r 2}$ are points' positions of axis $\mathrm{X}$ on upper and lower surfaces of the wall.

Or according to Snell's Law, a bivariate quartic equation can be expressed as Eq.2:

$$
\left\{\begin{array}{c}
\varepsilon_{1}\left(x_{r 1}-x_{a}\right)^{2}\left[d^{2}+\left(x_{r 2}-x_{r 1}\right)^{2}\right] \\
=\varepsilon_{2}\left(x_{r 2}-x_{r 1}\right)^{2}\left[z_{a}^{2}+\left(x_{a}-x_{r 1}\right)^{2}\right] \\
\varepsilon_{2}\left(x_{r 2}-x_{r 1}\right)^{2}\left[\left(z_{b}+d\right)^{2}+\left(x_{b}-x_{r 2}\right)^{2}\right] \\
=\varepsilon_{1}\left(x_{b}-x_{r 2}\right)^{2}\left[d^{2}+\left(x_{r 2}-x_{r 1}\right)^{2}\right]
\end{array}\right.
$$

where $x_{r 1}$ and $x_{r 2}$ are the two refract points' positions of axis $\mathrm{X}$ on the wall. Then we get the one-way time-delay by Eq.3:

$$
\begin{gathered}
\tau=\frac{1}{c}\left[\sqrt{\varepsilon_{1}} \cdot \sqrt{z_{a}^{2}+\left(x_{a}-x_{r 1}\right)^{2}}+\sqrt{\varepsilon_{2}} \cdot \sqrt{d^{2}+\left(x_{r 2}-x_{r 1}\right)^{2}}\right. \\
\left.+\sqrt{\varepsilon_{3}} \cdot \sqrt{\left(z_{b}+d\right)^{2}+\left(x_{b}-x_{r 2}\right)^{2}}\right]
\end{gathered}
$$

But the two dimensional searching method is relatively slow and solving the bivariate quartic equation is time-consuming.

\subsection{Fast time-delay calculation method (FTD)}

A new time delay calculation method (FTD) is presented. Total propagation path can be divided into three parts. The first part is the path in the wall; the other two parts are two paths at both sides. Because of the same environment outside the wall, we can confirm that incident angle at point $\mathrm{B}$ equals to refracting angle at point $C$. Defining the top surface of the wall as $z=0$, the starting point's coordinate is $\left(x_{a}, z_{a}\right)$, and the receiving point's coordinate is $\left(x_{b}, z_{b}\right), d$ is the thickness of the wall. The lateral distance between point $\mathrm{A}$ and point $\mathrm{B}$ is $l_{\text {total }}=\left|x_{b}-x_{a}\right| \quad$, and can also presented as $l_{\text {total }}=l_{\text {inner }}+l_{\text {outer }}$, where $l_{\text {total }}$ is the lateral distance in the wall, $l_{\text {outer }}$ is the lateral distance outside the wall.

The incident angle at point $\mathrm{C}$ is equal to the angle refract at point $\mathrm{D}$, so the ratio of the lateral distance and the vertical distance outside the wall is the same.

Then $l_{\text {outer }}$ can be divided into two parts, the distance up the wall and the distance down the wall:

$$
l_{\text {outer }}=l_{\text {up }}+l_{\text {down }}
$$

where $\quad l_{u p}=\frac{\left|z_{a}\right|}{\left|z_{a}\right|+\left|z_{b}+d\right|} l_{\text {outer }}, l_{\text {down }}=\frac{\left|z_{b}+d\right|}{\left|z_{a}\right|+\left|z_{b}+d\right|} l_{\text {outer }}$. According to Snell's Law, we can get Eq.5:

$$
\frac{l_{u p}^{2}}{l_{u p}^{2}+z_{a}^{2}} \cdot \frac{l_{\text {inner }}^{2}+d^{2}}{l_{\text {inner }}^{2}}=\frac{\varepsilon_{2}}{\varepsilon_{1}}
$$

where $\frac{\sin \theta_{i}}{\sin \theta_{t}}=\frac{\sqrt{\varepsilon_{2}}}{\sqrt{\varepsilon_{1}}}, \sin \theta_{i}=\frac{l_{u p}}{\sqrt{l_{u p}^{2}+z_{a}^{2}}}$, $\sin \theta_{t}=\frac{l_{\text {inner }}}{\sqrt{l_{\text {inner }}^{2}+d^{2}}}$.

In addition, another equation according to the geometric similarity relationship between the incident angle and the refracting angle can be expressed as Eq.6:

$$
l_{u p}=\frac{\left|z_{a}\right|}{\left|z_{a}\right|+\left|z_{b}+d\right|}\left(\left|x_{b}-x_{a}\right|-l_{\text {inner }}\right)
$$

A quartic equation about $l_{\text {inner }}$ can be deduced by two simultaneous equations Eq.5 and Eq.6:

$$
s_{4} l_{\text {inner }}^{4}+s_{3} l_{\text {inner }}^{3}+s_{2} l_{\text {inner }}^{2}+s_{1} l_{\text {inner }}+s_{0}=0
$$

where $s_{4}=c_{a}^{2}\left(\varepsilon_{2}-\varepsilon_{1}\right) \quad, \quad s_{3}=2 c_{a} c_{b}\left(\varepsilon_{2}-\varepsilon_{1}\right)$, $s_{2}=\left[\varepsilon_{2}\left(c_{b}^{2}+z_{a}^{2}\right)-\varepsilon_{1}\left(c_{a}^{2} d^{2}+c_{b}^{2}\right)\right] \quad, \quad s_{1}=-2 \varepsilon_{1} c_{a} c_{b} d^{2}$, $s_{0}=-\varepsilon_{1} c_{b}^{2} d^{2}, c_{a}=-\frac{\left|z_{a}\right|}{\left|z_{a}\right|+\left|z_{b}+d\right|}, c_{b}=\frac{\left|z_{a}\right| \cdot\left|x_{b}-x_{a}\right|}{\left|z_{a}\right|+\left|z_{b}+d\right|}$.

The lateral distance in the wall $l_{m}$ can be calculated by solving the simple quartic equation, and the time delay can be shown as Eq.8:

$$
\tau=\frac{l_{p}}{c}
$$

where

$$
\begin{aligned}
& l_{p}=\sqrt{\varepsilon_{1}} \sqrt{l_{u p}^{2}+z_{a}^{2}}+\sqrt{\varepsilon_{2}} \sqrt{l_{m}^{2}+d^{2}}+\sqrt{\varepsilon_{1}} \sqrt{l_{d o w n}^{2}+\left(z_{b}+d\right)^{2}}, \\
& l_{u p}=\frac{\left|z_{a}\right|}{\left|z_{a}\right|+\left|z_{b}+d\right|}\left(\left|x_{b}-x_{a}\right|-l_{m}\right), l_{\text {down }}=\frac{\left|z_{b}+d\right|}{\left|z_{a}\right|} l_{u p} \text {. }
\end{aligned}
$$




\section{Simulation experiment}

In this example, both sides are air, the relative permittivity of air and wall are $\varepsilon_{1}=1, \varepsilon_{2}=8$ respectively, the thickness of the wall is $0.5 \mathrm{~m}$, the coordinates position at starting point $\left(x_{a}, z_{a}\right)$ position is $(1,0.2)$, the imaging region is $x_{o} \in[-1,3], z_{o} \in[-3,-1.5]$.

Firstly, the accurate time-delay of the imaging region points calculated by traditional method can be shown as Fig.2 (a).The computation time is 7.3291s on the computer which has 2G RAM and Intel-Core CPU with $2.17 \mathrm{GHz}$ frequency. Next applying the fast time-delay calculation method (FTD), the computation time is $0.2726 \mathrm{~s}$, which only about 3.72 percent of the tradition method. The relatively error between the traditional method and fast calculation method is about $10^{-5}$, and the relatively error is zero in some imaging pixels as shown in Fig.2 (b).So, the FTD method is faster than traditional calculation, but the relatively error is very small.

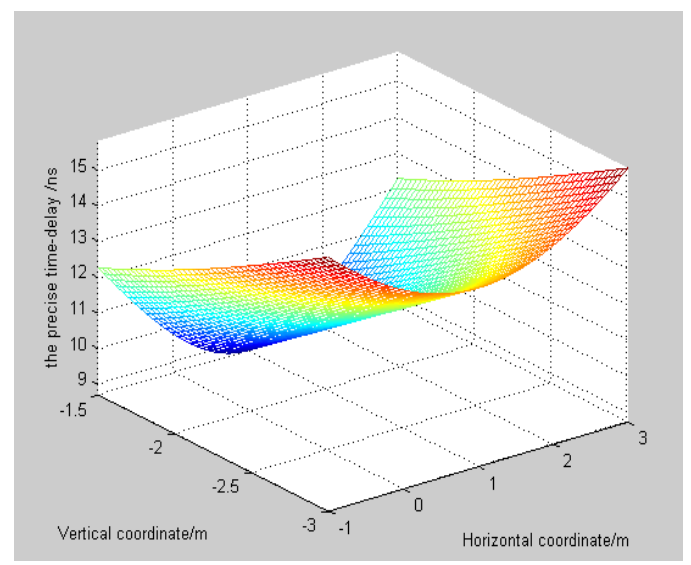

(a)time-delay calculated by traditional method

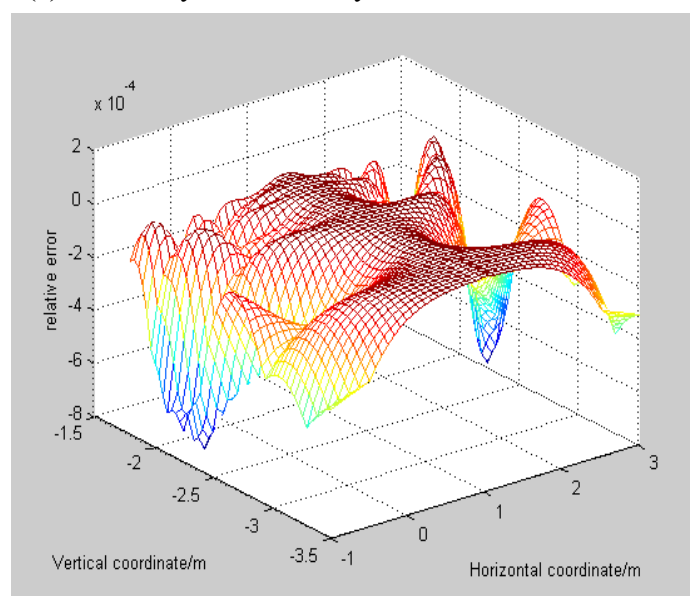

(b)relatively error between method and FTD method

Figure 2. The simulation experiment of FTD method

\section{Conclusion}

In this paper, a new fast time-delay calculation method is presented. By analyzing the electromagnetic waves propagate path, the lateral distance and total path; the time-delay can be got quickly. Simulation experiment shows the new algorithm is highly efficient and the error rate is low, and suitable for real-time positioning and image processing in TWR.

\section{Acknowledgement}

This work was financially supported by Science and Technology Project of Hunan Province (Grant No. 2015JC3043) and Research Fund of Shanxi Key Laboratory of Electronic Information System Integration (Grant No.2015AA018).

\section{References}

1. Jol H M.Ground Penetrating Radar Theory and Applications[J]. Ground Penetrating Radar Theory \& Applications, (2009):509-524.

2. Dogaru T, Le C. SAR Images of Rooms and Buildings Based on FDTD Computer Models.[J]. IEEE Transactions on Geoscience \& Remote Sensing, (2009), 47(5):1388 - 1401.

3. Ahmad F, Amin M G, Kassam S A. Synthetic aperture beamformer for imaging through a dielectric wall[J]. IEEE Transactions on Aerospace Electronic Systems,( 2005), 41(1):271-283.

4. Johansson E M, Mast J E. Three-dimensional ground-penetrating radar imaging using synthetic aperture time-domain focusing[C]/SPIE's 1994 International Symposium on Optics, Imaging, and Instrumentation. International Society for Optics and Photonics, (1994): 205-214.

5. Rappaport C M. Accurate Determination of Underground GPR Wavefront and B-Scan Shape From Above-Ground Point Sources[J]. IEEE Transactions on Geoscience \& Remote Sensing, (2007), 45(8):2429-2434.

6. Wen T L, Yu J S. An Improved Iteration Back Projection Imaging Technique for Surface Penetrating Radar Applications. Applied Mechanics \& Materials, (2013), 1509-1513.

7. Chen X, Xue S, Feng D, et al. A new wall clutter reduction strategy for through wall radar imaging[C]//Signal Processing (ICSP), 2014 12th International Conference on. IEEE,(2014): 21022105.

8. Protiva P, Mrkvica J, Machac J. Estimation of Wall Parameters From Time-Delay-Only Through-Wall Radar Measurements[J]. IEEE Transactions on Antennas \& Propagation, (2011), 59(11):4268-4278. 